\title{
TTR
}

Traduction, terminologie, re?daction

\section{Dans la ruine de Babel : poésie et traduction chez Paul Celan}

\section{Alexis Nouss}

Volume 9, numéro 1, 1er semestre 1996

Le festin de Babel

Babel's Feast

URI : https://id.erudit.org/iderudit/037237ar

DOI : https://doi.org/10.7202/037237ar

Aller au sommaire du numéro

\section{Éditeur(s)}

Association canadienne de traductologie

ISSN

0835-8443 (imprimé)

1708-2188 (numérique)

Découvrir la revue

Citer cet article

Nouss, A. (1996). Dans la ruine de Babel : poésie et traduction chez Paul Celan. TTR, 9(1), 15-54. https://doi.org/10.7202/037237ar

\section{Résumé de l'article}

Dans la ruine de Babel : poésie et traduction chez Paul Celan — Paul Celan, l'un des plus grands poètes de langue allemande du XXe siècle, en est aussi l'un des plus grands traducteurs. Sur les cinq tomes de ses Oeuvres complètes chez Suhrkamp, deux volumes sont consacrés à ses traductions, en majorité de la poésie, couvrant sept langues et près d'une cinquantaine d'auteurs. Le présent article tente de montrer qu'une même poétique anime les deux gestes d'écriture et de traduction et qu'une telle entreprise doit se comprendre par sa dimension éthique. Il s'agit pour Celan de recréer un langage humain, ouvert à l'altérité, répondant à cette sombre historicité qui est de vivre après Auschwitz.

Tous droits réservés ( $\mathrm{C}$ TTR: traduction, terminologie, rédaction — Les auteurs, Ce document est protégé par la loi sur le droit d’auteur. L’utilisation des 1996 d'utilisation que vous pouvez consulter en ligne.

https://apropos.erudit.org/fr/usagers/politique-dutilisation/ 


\section{Dans la ruine de Babel : poésie et traduction chez Paul Celan ${ }^{1}$}

\section{Alexis Nouss}

Ruine à entendre aux deux sens : ce qui resterait encore de Babel, compris comme la possibilité d'une parole, plurielle en ce qu'elle est parole, support d'entente mutuelle et d'interprétation réciproque mais aussi l'effondrement de Babel, l'affirmation que la parole est désormais impossible puisque la barbarie contemporaine l'a privée de tout support ontologique et de toute relation au réel. Ce qui est décrit là ne sacrifie pas à une esthétique du tragique, un pathos du désastre, mais dessine un horizon éthique et herméneutique. Résister sans succomber à l'aveuglement nihiliste mais au contraire accepter l'histoire dont nous sommes à la fois acteurs et héritiers pour y fixer notre regard. Ruine résume alors l'impression que provoque la vision de vestiges : celle d'une interruption dans la temporalité vécue comme continuité, d'une faille dans la durée, la table encore dressée que l'irruption volcanique ou le naufrage du navire ont par exemple saisie pour les temps à venir ${ }^{2}$. Scission chronologique,

1. Cet article s'inscrit dans le cadre d'une recherche subventionnée par le CRSH du Canada. Une version différente est panue dans les Actes du colloque Paul Celan (1995), Éditions Est-Ouest internationales, 1996.

2. L'image archéologique qu'utilise Freud dans la description du psychisme et du travail analytique se comprend de la même manière. Par ailleurs, à partir de son emploi chez Baudelaire 
fracture de la chrono-logie : « crevasse » ou " cicatrice » du temps selon les métaphores de Celan ${ }^{3}$. Pour ce qui est de l'histoire de notre siècle, inutile de nommer cette césure, ne serait-ce que parce qu'elle marque la limite de toute nomination : « ce qui s'est passé ", ainsi Celan désignait-il (de-signait-il) l'horreur des camps nazis.

Ce qui ne signifie pas la cessation de cette temporalité mais au contraire l'invitation à sa reprise. Précieuse ici l'image du pêcheur de perles employée par Hannah Arendt pour qualifier la pensée et la méthode d'un Walter Benjamin, tourné vers le passé comme réceptacie de moments présents cristallisés et en attente de remonter à la surface de l'aujourd'hui. La poésie de Celan se veut un tel témoignage : les voix éteintes sont ravivées dans et pour le dialogue, non dans la seule écoute, résurrection artificielle de l'historicisme. "Ihr/Sinkenden, hört/auch uns" ; "Vous/qui sombrez, entendez-nous/aussi. » (1993, pp. 10-11)

"WORTAUFSCHUTTÜNG, vulkanisch,/meerüberrauscht" ; " LEVÉE DE MOTS, volcanique,/recouverte par le grondement de la mer. " $(1987$, p. 104).

\section{Paul Celan, poète et traducteur}

L'ange de la mort, qui se nomme Samael dans certaines légendes, et dont on raconte que Molse lui-même dut l'affronter, est le langage. L'ange nous annonce la mort - et que fait d'autre le langage? (G. Agamben, Idée de la prase)

la ruine devient emblématique d'une certaine pensée de la modernité (voir A. Nouss, 1995b).

3. Voir infra.

4. Les indications bibliographiques entre parenthèses dans le corps du texte renvoient aux traductions des ceuvres de Celan figurant dans les références à la fin de l'article. En leur absence, la traduction est mienne. 
Si la poétique de Celan répond ainsi à la philosophie de Benjamin, toutes deux illisibles sans considérer l'histoire qui les a mises au jour, elles font signe vers la traduction, centrale dans les deux cuvres, en ce que celle-ci se présente comme l'instauration d'un dialogue au présent entre des textes à la fois séparés et unis par la temporalité.

La ruine de Babel est au cceur de l'œuvre de Paul Celan. Parce qu'elle en est un des thèmes essentiels mais aussi parce qu'il a su montrer et porter jusqu'à un point extrême, point d'incandescence, point de fusion, ce que la pensée peut aujourd'hui penser de la langue, ce qu'il nous reste à penser de la langue et ce qu'il y a d'impuissance à penser en dehors de la langue.

Dans sa poésie, de manière exemplaire, la langue (allemande) travaille d'autres langues, est travaillée par elles et travaille son propre deuil. En ce sens, la traduction, opération langagière fonctionnant sur l'écart, la distance, l'absence et la perte, s'offre comme un mode d'énonciation permettant de saisir ce qui est en jeu dans l'écriture celanienne. En retour, une telle application herméneutique jette un précieux éclairage sur la nature même de la traduction.

Paul Celan est l'une des grandes voix poétiques du siècle, le plus grand poète de langue allemande depuis Rilke, proclame-t-on souvent, citant s'il le faut Heidegger ou Adorno à l'appui. Mais l'énoncé, outre qu'il a désormais statut de lieu commun, s'avère peu signifiant puisqu'il ignore l'effet de césure, de syncope, de retardement', prémentionné au profit d'une factice continuité de la tradition littéraire qui échapperait aux bouleversements de l'histoire. Pour ne pas succomber à ce que Meschonnic a baptisé "l'effet Celan », le passage de Celan à "Celan " (Meschonnic, 1990, p.

5. "Lesenstationen im Spätwort", " Stations de lecture dans le mot retardé" (Lichtzwang). Voir sur ce point notre article « La demeure de la lettre» à paraître en 1996 dans un numéro d'Études littéraires consacré à " Littérature et judéité ". 
170) qui autorise à ne pas tirer les conséquences de sa poétique, en procédant par sacralisation et poétisation, idéologisation et mythologisation, il est impérieux de fonder dans l'histoire cette ceuvre : « La tension vers le mutisme dans l'écriture de Celan est enracinée dans son vivre le langage, vivre l'histoire. [...] C'est une aventure par le langage, elle s'est fait ses propres lois avec son histoire. " (Meschonnic, 1986, p. 372) On peut au demeurant repérer une sensibilité critique nord-américaine insistant sur le contexte historique de la poésie celanienne alors qu'un certain courant européen aurait tendance à déhistoriciser l'œuvre en la tirant du côté de Heidegger.

Paul Celan, Paul Antschel de son nom patronymique, est né à Czernowitz, en Roumanie, en 1920. Il commence des études de médecine à Tours en 1938 mais doit retourner à Czernowitz en 1939 à cause de la guerre. Il entreprend alors des études de langues romanes. La ville est occupée par les nazis en 1941. Ses parents sont déportés en 1942. Lui est interné dans un camp de travail. Il retourne à Czernowitz en 1944, reprend ses études et travaille à partir de 1945 à Bucarest comme lecteur et traducteur pour une maison d'édition. Il quitte la Roumanie en 1947 pour Vienne puis pour Paris en 1948 où il s'installe jusqu'à la fin de sa vie. Il obtient une licence ès lettres en 1950 et devient lecteur d'allemand à l'École Normale Supérieure à partir de 1959. Son œuvre poétique, douze recueils, est publiée de 1948 à 1971 et est très vite reconnue au premier rang de la poésie occidentale du siècle.

Il est traducteur, vers l'allemand : du russe (Blok, Mandelstam, Essenine, Evtouchenko, Slutschewski, Chlebnikov), de l'anglais (Shakespeare, Marianne Moore, Andrew Marvell, Emily Dickinson, Robert Frost, Alfred Edward Housman, John Donne), de l'italien (Ungaretti), du roumain (Gellu Naum, Virgil Teodorescu, Tudor Arghezi), du portugais (Fernando Pessoa - sous ses divers hétéronymes - en collaboration avec Edouard Roditi), de l'hébreu (David Rokeah), du français (Pablo Picasso, Jean Cayrol, Rimbaud, Valéry, A. Du Bouchet, Supervielle, Char, Michaux, J. Dupin, Breton, A. Césaire, H. Pastoureau, B. Péret, Apollinaire, Artaud, Desnos, Nerval, Éluard, Mallarmé, Baudelaire, Maeterlinck, Jean 
Daive). Parfois des ceuvres entières, parfois un texte unique, quelquefois de la prose, en majorité de la poésie, du XVI' siècle au $\mathrm{XX}^{\mathrm{e}}$. Dans ses cuvres complètes publiées par Suhrkamp en cinq volumes, les deux derniers recueillent uniquement ses traductions et le dernier uniquement les traductions du français. Et l'éditeur nous informe que sont absentes des cuvres complètes une vingtaine d'autres traductions, de l'anglais et du français, plusieurs articles mais aussi le Jabberwocky de Lewis Caroll et deux Maigret de Simenon. Sont mentionnées également trois traductions du russe vers le roumain publiées sous d'autres pseudonymes. Ne sont pas signalées en revanche des traductions de Kafka faites en roumain par Celan dans sa jeunesse.

Un des plus grands poètes du siècle, il en est sans doute aussi l'un des plus grands traducteurs, et le lien est signifiant : les deux pratiques ne sont pas autonomes et il s'agit d'examiner quelle en est la commune poétique ${ }^{6}$.

En avril 1970, il se jette dans les eaux de la Seine. Son itinéraire, " vie mutilée » pour reprendre l'expression d'Adorno, résume les déchirures d'une identité européenne moderne brisée par le mal totalitaire.

Lire Celan, c'est comprendre que dans l'écriture, le rapport entre les langues, dont la traduction est a la fois un des modes et le modèle même, ainsi que le rapport de la langue à elle-même, autre

6. L'histoire littéraire montrant qu'un grand nombre de poètes furent et sont aussi traducteurs, s'offre en cette convergence une riche voie de recherche pour la traductologie. Des auteurs đu XVI" siècle jusqu'à ceux de la modernité (commençant avec Nerval, Baudelaire ou Mallarme), cette approche renouvellerait les analyses sociologisantes des tenants du polysystème ou des descriptive studies situant la traduction littéraire dans le cadre de la littérature en général. Le lien entre écriture et traduction a pourtant suffisamment été théoriquement établi par Meschonnic (voir notamment Pour la poétique II). 
forme de ce rapport, n'ont de sens qu'interrogés en regard de l'historicité du projet.

Celui de Celan visait à l'établissement d'un geste langagier d'avant la parole, neutralité nécessaire à l'accueil éthique de l'autre et à la restauration de l'humain', où donc la juxtaposition des langues, loin de créer une plénitude, creuse le verbe vers l'absolu d'un vide dont l'autre nom est silence. Comme l'écrit Jabès à propos de la langue de Celan, langue des bourreaux et des victimes, langue " dépossédée et retrouvée " : "Détruíre ce qui cherche à être dit, avant de le dire ; comme si le silence, seul, avait maintenant droit de cité : ce silence d'avant et d'après les mots, ce silence entre les mots, entre deux langues, dressées l'une contre l'autre et, cependant, promises à un même destin. " (Jabès, 1990, p. 18)

L'historicité de la langue aujourd'hui - son usage et sa nature -, l'historicité d'une réflexion sur la langue, $m$ 'amène à poser ceci, à partir de Paul Celan : nous vivons une ère post-langagière. Nous parlons, certes, mais une langue d'après le langage. Une langue privée de ce qui fait d'elle un mode langagier, privée de sa dignité ontologique. Paradoxe que d'articuler en un code un message proclamant la fin de ce code, son effacement ? Comment puis-je dire que je ne parle pas dans l'énoncé et l'énonciation de cette parole ? Comment le bruit peut-il manifester le silence ? Comment la lumière peut-elle désigner la ténèbre ? Comment le fini peut-il contenir l'infini ? Comment l'humain peut-il nommer Dieu ? Tous ces exemples pour montrer que la question n'est pas, en son genre, si inhabituelle, qu'elle reprend une rhétorique attestée. $\dot{A}$ cette interrogation, on a répondu : le discours mystique, pour dire le transcendant dans l'immanent ou la peinture abstraite, pour dire l'irreprésentable dans la figuration. Et, pour nous rapprocher du domaine langagier, tout un courant de la littérature moderne ne fait

7. On reconnaîtra aisément ce que cette description doit à la pensée d'Emmanuel Lévinas. 
que décliner la perte du langage, trouvant son inspiration dans ce souffle absent ${ }^{8}$.

Une langue d'après le langage, une langue pour dire l'aprèslangage. Pas plus aporétique que la notion de post-histoire, si courante désormais. En différentes versions : hégélianisme revu et corrigé (la fin de l'histoire à la Fukuyama : triomphe du modèle [américain] démocratique et libéral), post-modernité (disparition des grands récits structurant une conception de l'histoire comme progrès et progression) ou une troisième approche sur laquelle je préfere m'attarder : pensée au sein de l'École de Francfort, notamment exprimée par Adorno et Benjamin, cette critique, pour sauver l'autonomie de l'individu, s'attaque à l'histoire considérée comme totale et homogene et au monde comme monde de domination et de réification. L'histoire n'est plus une, elle est fragmentée, éclatée, faite de ruptures et de reprises. Elle n'est plus analogue au courant fluvial dont le flot s'écoule impérieux mais serait davantage métaphorisée sous les espèces des vagues océanes se succédant rhapsodiquement. L'avant et l'après perdent leur logique de continuité pour acquérir des dynamiques autonomes et ni l'un ni l'autre n'ont statut de supériorité. Le nazisme a ainsi introduit une rupture telle dans l'aventure humaine que l'humanité, quoiqu'encore en défaut de conscience, est entrée dans une nouvelle ère. D'où la célèbre déclaration d'Adorno : " [...] écrire un poème après Auschwitz est barbare, et ce fait affecte même la connaissance qui explique pourquoi il est devenu impossible d'écrire aujourd'hui des poèmes " (Adorno, 1986, p. 23), dont, on le verra, la poésie de Celan n'est pas un démenti mais une illustration, voire une confirmation.

Une langue, donc, d'après le langage pour une historicité d'après l'histoire. Ce que dit George Steiner en posant bien les conséquences métaphysiques de son propos :

8. Voir M. Blanchot (1988) et, plus récemment, R. Robin (1993). 
Il se peut que l'univers d'Auschwitz (car c'est ce qu'il a été) désigne ce royaume d'une bestialité potentielle (désormais réalisée), ou plutôt d'abandon de l'humain et de régression à la bestialité, qui à la fois précède le langage, comme c'est le cas chez l'animal, et qui vient après le langage, comme cela a lieu dans la mort. Auschwitz signifierait à l'échelle historique, collective, la mort de l'homme en tant qu'organisme parlant, rationnel, "rêveur d'avenir " (le zoon phonanta de la philosophie grecque). Les langages que nous parlons désormais sur cette planète corrompue et suicidaire sont post-humains. [...] Ils retentissent de vide, avec une intensité que les médias électroniques rendent encore plus manifeste et barbare. Là où le langage est encore humain, dans le sens fondamental de ce mot, il est parlé par des survivants, des gens qui se souviennent et des fantômes. Sa musique hantée est celle des braises qui continuent à crépiter dans la cendre refroidie d'un feu mort. (Steiner, 1987, p. 17)

Que l'œuvre de Paul Celan relève de part en part de cette langue post-langagière se montre de sa facture et de son thème. Les deux à considérer ensemble, évidemment, et qui forment une poétique. Innombrables les poèmes où figurent les signifiants " langue ", " silence ", " mot $" .$. et la critique celanienne a justement établi combien la poésie de Celan choisit comme objet et projet la possibilité de sa propre écriture.

Sa langue a été largement commentée : sa pureté, son âpreté, ses tournures caractéristiques (dislocations ou distorsions syntaxiques et morphologiques, compositions lexicales), l'emploi de lexiques archaiqques ou techniques, la dislocation typographique. Quant à la lecture, il n'est que banal de dire qu'elle n'est pas facile. On qualifie rapidement sa poésie d'hermétique. Faut-il confondre inintelligibilité et hermétisme ? Peter Szondi séparait les deux. Adorno insiste sur la nature sociale et la fonction stratégique de l'hermétisme :

La conscience réifiée qui, dans l'intégration de la société industrielle avancée, s' intègre à travers les membres de celle-ci, est incapable de percevoir l'essentiel des poèmes, et n'y voit 
que leurs contenus thématiques et leurs prétendues valeurs d'information. Sur le plan artistique, les hommes ne peuvent plus, d'ailleurs, être atteints que par le choc visant ce que l'idéologie pseudo-scientifique appelle communication. L'art, quant à lui, n'est intègre que lorsqu'il ne joue pas le jeu de la communication. (Adorno, 1989, pp. 405-406)

Ce qui, d'emblée, énonce un intéressant problème traductologique : comment traduire alors ce qui se donne dans le secret, dans la non-communication si là est le trait essentiel de l'art authentique aujourd'hui ? Nous y reviendrons plus loin mais disons pour l'heure que cette réflexion d'Adorno fait écho au début si controversé de " la Tâche du traducteur " de Benjamin : "Une traduction qui [...] veut communiquer ne saurait transmettre que la communication - donc quelque chose d'inessentiel. " (Benjamin, 1971, p. 261)

Lisant Celan, on ne le comprend certes pas immédiatement mais cette incompréhension est utile car elle nous invite à repenser le statut de la compréhension et de la communication, le « sens du sens » comme dirait Steiner qui a su décrire le soupçon sur le mot et le langage né à la fin du $\mathrm{XIX}^{\circ}$ siècle et que le $\mathrm{XX}^{\circ}$ a tragiquement confirmés, culture du mutisme ou de l'aphasie dans laquelle s'inscrit la langue de Celan. La difficulté de compréhension est un appel à l'effort d'intellection et à une compréhension d'autant plus profonde, une invitation à la responsabilité du lecteur, à un engagement éthique de sa part qui prend sens du contexte historique de sa lecture. Pseudo-hermétisme de Celan qu'il efface par sa phrase à l'éditeur Hans Bender en 1960 : " Je ne vois en principe aucune différence entre un serrement de mains et un poème. " (Broda, 1986, p. 109)

Sur ce point la traduction n'a rien à perdre. Contrairement à l'idéologie encore régnante en matière de traductologie, la traduction n'est pas congénitalement et consubstantiellement liée à la compréhension. Au contraire. Une formule, faute de place, le résumera : moins on comprend, plus on traduit. 
Steiner décrit ainsi la langue de Celan en la situant dans son historicité et dans son lien à la traduction :

Épaulé par ses traductions à partir du russe, du français de l'anglais, il est en mesure, en décalant l'allemand, de la placer en position d'étrangeté bénéfique. Il a la possibilité de l'arborer avec un détachement clinique, comme un matériau brut qui luj revient inévitablement tout en demeurant contingent et susceptible d'hostilité. Sa propre poésie est, dans sa totalité, traduction en allemand. Au cours de laquelle la langue-cible se trouve chassée de chez elle, démantelée, particulariséejusqu'aux limites de la non-communication.C'est alors un " méta-allemand " décapé de sa crasse historique et politique, et par là même utilisable, après l'holocauste, par une voix profondément juive. (Steiner, 1978, p. 359)

Pour Meschonnic, "Celan fait une contre-langue dans la langue, pour se situer dans les contradictions de son histoire, leur faire face sur le terrain même de la plus grande aliénation." (Meschonnic, 1986, pp. 375-376)

Méta-allemand ou contre-langue, les deux notions se rejoignent dans cette idée d'une altérité au sein mềme de l'identité langagière. Se retrouve ici la dynamique antagoniste ou dialogique qui, chez Celan, se symbolise dans le signifiant " gegen 》: le "Gegenwort " du " Discours du Méridien " ou le contre-jour, Gegenlicht, qu'il a utilisé comme titre à deux reprises. La langue de Celan n'offre pas seulement le chatoiement de langues différentes (hébreu, espagnol, français dont des mots apparaissent tels des éclats insérés dans l'allemand des poèmes qui, de par sa facture, se présente déjà comme une mosaïque davantage que comme un tissu) s'enrichissant mutuellement mais elle révèle aussi ce travail où une langue devient autre à elle-même. " Ma langue est une autre ", pourrait-on dire, paraphrasant Rimbaud. L'écriture a été théorisée comme la pratique d'une langue étrangère où l'acte même d'écrire est déjà un acte d'altération, d'altérité. " On parle dans sa propre langue, on écrit en langue étrangère ", écrit Sartre (1972, p. 135), comme en écho d'un passage de Proust qui dans le Contre Sainte- 
Beuve développait ce thème en l'amplifiant et le plaçant délibérément dans le cadre du processus traduisant : " Les beaux livres sont écrits dans une sorte de langue étrangère. Sous chaque mot chacun de nous met son sens ou du moins son image qui est souvent un contre-sens. Mais dans les beaux livres, tous les contresens qu'on fait sont beaux. "(Proust, 1971, p. 305) Deleuze thématise cet écart et cet écartement de sens en faisant de l'écrivain un " bègue de la langue ", métaphore, entre autres, de la minorisation langagière propre aux grands écrivains, Kafka, Beckett :

Ce qu'ils font, c'est plutôt inventer un usage mineur de la langue majeure dans laquelle ils s'expriment entièrement : ils minorent cette langue, comme en musique où le mode mineur désigne des combinaisons dynamiques en perpétuel déséquilibre. Ils sont grands à force de minorer : ils font fuir la langue, ils la font filer sur une ligne de sorcière, et ne cessent de la mettre en déséquilibre, de la faire bifurquer et varier dans chacun de ses termes suivant une incessante modulation. [...] Autant dire qu'un grand écrivain est toujours comme un étranger dans la langue où il s'exprime, même si c'est sa langue natale. $\dot{A}$ la limite, il prend ses forces dans une minorité muette inconnue qui n'appartient qu'à lui. C'est un étranger dans sa propre langue : il ne mélange pas une autre langue à sa langue, il taille dans sa langue une langue étrangère et qui ne préexiste pas. Faire crier, faire bégayer, balbutier, murmurer la langue en elle-même. (1993, p. 138)

Métamorphose qu'illustre parfaitement l'allemand de Celan mais il nous fait entendre aussi une étrangeté à un autre niveau. La langue peut devenir étrangère à celui qui la parle et qui n'a pourtant rien d'autre en partage. Celan témoigne d'une double césure, celle de l'individu par rapport à sa langue et celle de l'individu par rapport à son co-locuteur, double blessure infligée par l'histoire : " Elle, la langue, fut sauvegardée, oui, malgré tout. Mais elle dut alors traverser son propre manque de réponses, dut traverser un mutisme effroyable, traverser les mille ténèbres des discours meurtriers. " (" Discours de Brême ", Poèmes, 1987, p. 16) Non 
plus seulement le sujet en exil de la langue, thème cher à la modernité, d'Hofmannstahl (Lettre de Lord Chandos) à Artaud, mais la langue en exil de ses locuteurs, une langue perdue, comme une mère, comme une patrie :

"[...] wie heisst es, dein Land/hinterm Berg, hinterm Jahr? [...] das Dreijahreland deiner Mutter, das war es,/das ists,/er wandert überallhin, wie die Sprache,/wirf sie weg, wirf sie weg/dann hast du sie wieder [...]. " ; " [...] comment s'appelle-t-il, ton pays/derrière les monts, derrière l'année ? [...] le pays-des-trois-ans de ta mère, c'était lui,/c'est lui,/il émigre partout, comme la langue,/ rejette-la, rejette-la,/et tu l'auras de nouveau [...]."

" [...] in das reich,/in der Reiche/weitestes, in /den Grossbinnenreim/jenseits/der Strummvolker-Zone, in dich/ Sprachwaage, Wortwaage, Heimat-/waage Exil.» ; " [...] dans le royaume,/dans le plus vaste/des royaumes, dans/la grande rime intérieure/au-delà de/la zone-des-peuples-muets, en toi/ balance de la langue, de la parole, du lieu natal,/balance exil. " (1979a)

Celan, s'appuyant sur l'histoire de son siècle, de notre siècle, tire les conséquences de la chute d'une langue (l'allemand mais aussi bien toute langue, les exemples actuels ne manquent pas) devenue incapable d'encore assurer son rôle. De l'historique il tire une nouvelle ontologie du langage. On ne peut plus faire confiance au langage quand celui-ci a permis la parole des bourreaux. D'où ce qui passe pour l'obscurité de sa poésie mais qui n'est que l'opacité du langage aujourd'hui, tel qu'il est devenu depuis le passage par le gouffre. D'où aussi l'appel au traduire comme mode fondamental du langage. Übersetzung : faire passer au-delà puisqu'aussi bien la langue allemande, l'originelle, la pure, la non-corrompue, la maternelle, est restée de l'autre côté, autre côté de l'histoire et du gouffre, du côté de l'avant. Le deuil de la langue, cependant, oblige à trouver une langue du deuil, qui va rendre possible ce travail.

[...] je pense que c'est depuis toujours une espérance du poème, de parler, avec ce langage justement, comme si c'était 
d'ailleurs - non, je ne peux plus utiliser ce mot désormais, comme si c'était au nom d'un autre - qui sait, peut-être au nom d'un tout autre. [...] peut-être même une coïncidence entre ce " tout autre" - une expression usuelle pour désigner ce qu'on ne sait pas - et, non plus lointain, tout proche, un " autre ", est-elle pensable - toujours et encore pensable. [...] le poème est au plus fort quand il est au bord de lui-même ; c'est de là qu'il appelle, mais il ne peut plus s'y tenir qu'en s'arrachant sans cesse de son déjà-plus vers son encore. (« Le Méridien », 1979b)

" De son déjà-plus vers son encore », n'est-ce pas là, dans ce que dit Celan de la poésie aujourd'hui, n'est-ce pas là la phénoménologie du traduire ? Car, tragiquement, si l'historicité de l'écriture celanienne - une langue d'après le langage - éclaire à nos yeux le processus traductif, c'est qu'une telle poetique trouve dans la traduction sa dynamique même et son mode d'existence. Dans la langue perdue et retrouvée mais conservant la trace de sa perte - condition même du langage aujourd'hui - se dessine l'être du traduire, la traduction esquissant en retour la tension ontologique désormais attachée au langage.

De la même langue, Celan fait une autre langue, et qu'il le fasse par le biais de la poésie ne saurait étonner puisque le verbe poétique trouve là, de manière générale, sa légitimité et sa fonction. De même, en traduction, le texte d'arrivée n'est pas le double du texte de départ, il s'inscrit contre (aux deux sens du terme en français et dans le même rapport que conscient et inconscient dans la pensée freudienne) l'original. La traduction affirme alors ce qu'est la vérité du langage qui est la liberté du langage : la possibilité de dire la même chose (infirmant le concept même du même) d'une autre manière, ce qui prouve qu'il n'y a jamais la même chose, qu'une chose n'est jamais mếme (par le sujet et dans l'histoire). Tel est le propre de l'humain et cela se montre $a$ contrario des scénarios in-humains - mais historiques - qui, eux, tendent vers une langue de pierre (de bois, dit-on aussi), d'où la minéralité hantant la poésie de Celan, qui en vient ou qui en sort (elle s'écrit à partir de là et, dans ce mouvement, s'en échappe), minéralité qui équivaut à la 
mort, comme l'a remarqué Adorno. La traduction, dans cette perspective, perd son statut de genre d'écriture pour devenir un mode d'écriture, un modèle poétique.

Lorsque Philippe Lacoue-Labarthe voit s'opérer dans la poésie de Celan la " substitution de l'altérité à l'étrangeté », on peut reconnaître le mouvement et la loi de la traduction : ce qui était lointain devient proche sans que la proximité n'abolisse l'identité propre des deux textes. La relation est métonymique, non métaphorique, malgré l'étymologie, à l'encontre des théories de la traduction souscrivant à une logique de l'effacement (par le texte d'arrivée annulant le texte de départ). Il y a déplacement, non remplacement (Celan n'est-il pas, avant tout, une « personne déplacée ", comme Kafka, comme Benjamin, comme Perec ?). Benjamin qui proposait en esthétique une définition de l'" aura ", marquant l'authenticité de l'cuvre d'art, dans la dialectique entre le proche et le lointain, caractérise la teneur sémantique des grandes traductions : " [...] le sens n'est touché par le vent du langage qu'à la manière d'une harpe éolienne " (Benjamin, 1971, p. 275). Peter Szondi utilise la distinction articulée par Jakobson entre métaphore et métonymie pour conclure son analyse du processus traductif de Celan quant au sonnet 105 de Shakespeare qui de la « constance » thématisée dans l'original fait la matière et la manière même du poème. Et ce faisant, il rapproche écriture et traduction chez Celan dans une même poétique, précisément la poétique de la " fonction poétique " telle que Jakobson la définit : la projection du principe

9. Significativement, c'est dans le poème « Lob der Ferne ", "Louange du lointain ", que figure ce tercet exprimant au plus juste la dynamique traductive brouillant l'opposition ontologique entre le même et l'autre, le proche et le lointain : "Schwärzer im Schwarz, bin ich nackter./Abtrünnig erst bin ich treu./Ich bin du, wenn ich ich bin. " "Plus noir dans le noir je suis plus nu./Infidele seulement je suis fidèle./Je suis tu quand je suis je. " (1987, p. 69) Il y aurait lieu d'analyser ce brouillage à la lumière des développements de Benjamin sur l'aura et de Lévinas sur la proximité. 
d'équivalence de l'axe sélectif sur l'axe combinatoire. Mais pourquoi Szondi ajoute-t-il : « La définition de Jakobson ne décrit pas un poème, mais nomme le principe suivant lequel se règle l'usage de la langue poétique, dans le sens strict du terme. Ce principe ne peut jamais devenir complètement la réalité poétique, si du moins le poème ne veut pas être tautologique, s'il doit dire quelque chose " (Szondi, 1982, p. 159) ? Outre que tout poème, si obscur soit-il, ne peut entièrement sombrer dans la tautologie puisque l'usage de la langue empêche l'autisme sémantique - par l'intervention d'un sujet lecteur -, il nous semble, au contraire, que le « devoir dire " échappe totalement à la nature et à la fonction du poème celanien. C'est précisément à propos de la traduction que Benjamin insiste sur la non-intentionalité de l'œuvre d'art et sur la fonction non-communicante de la traduction. Et il présente la traduction non comme un medium d'information mais comme cette opération langagière destinée à révéler la différence et la complémentarité des modes d'expression langagiers. Le rapport traductif illumine l'essence de langue des langues : ce qu'elles se disent l'une à l'autre - et ce qui est ainsi donné à entendre au lecteur -, c'est qu'elles sont langues et, en tant que telles, recélant la " semence de pur langage ", « die reine Sprache ", " cette langue de vérité [qui] est le véritable langage ", langue de vérité et vérité de la langue. Vérité de la langue qu'il définit comme le langage se communiquant lui-même dans " Sur le langage en général et sur le langage humain 》.

De même la langue de la poésie de Celan ne cherche qu'à être langue, dernier recours dans le désastre du monde :

Sprich auch du,/sprich als letzter,/sag deinen Spruch./Sprich -/Doch scheide das nein nicht vom Ja./Gib deinem Spruch auch den Sinn:/gib ihm den Schatten//Gib ihm Schatten genug,/gib ihm so viel,/also du um dich verteilt weisst zwischen/Mittnacht und Mittag und Mittnacht./Blicke umher:/sich: wie's lebendig wird rings -1 Beim Tode/Lebendig!/ Wahr spricht, wer Schatten spricht. 
Parle toi aussi/parle en dernier,/dis ta parole.//Parle-/Mais sans séparer le non du oui./Donne aussi le sens à ta parole:/donnelui l'ombre.//Donne-lui assez d'ombre, donne-lui autant/que tu en sais partagée autour de toi entre/minuit et midi et minuit.//Regarde tout autour:/vois comme ce qui t'entoure devient vivant -/Près de la mort ! Vivant !/Qui parle l'ombre parle vrai. (1991; tr. modifiée)

Le dire qui reste tel et ne se fige pas en dit ne meurt pas. Vie en vérité, contre et malgré la mort. L'ombre comme vraie vie alors que pour le langage le dit serait la mort (et le parallèle a été établi de l'arbitraire du système signifiant et de la violence mortifère nazie par W. Menninghaus dans Paul Celan: Magie der Form). Le langage ne s'efface pas en dit. Il demeure dans l'ombre si l'on définit la signification comme clarté. « Entre minuit et midi et minuit » : de la nuit à la nuit, entre deux nuits, la chronologie juive dessine l'espace de la vérité langagière aujourd'hui. Et Lévinas a montré comment la clarté peut appartenir à la violence d'un logos totalisateur et ramenant au même, une intellection qui est négation de l'autre. L'ombre est ce qui permet au sujet de naître dans le langage, là où l'histoire a tenté de le faire disparaître. Ombre et nuit, récurrentes dans la poésie de Celan, qui écrit dans Lichtzwang :

"Sprache, Finster-Lisene ", " parole : lisière de l'obscur " (1987a) ; " Doch konnten wie nicht/hinüberdunkeln zu dir/er herrschte/Lichtzwang." " Mais nous ne pouvions pas/ ténébrer vers toi:/ il régnait/la contrainte de lumière. » (1989)

L'obscurcissement est le voilement de l'évidence langagière, l'invitation à la reprise, à la nouvelle version, à la traduction ${ }^{10}$. La nuit n'est ni le neutre heideggerien ni le contraire du jour ; c'est un espace autre où l'autre ne peut être saisi, ramené à du même, mais où il se déploie hors d'atteinte de la main ou de l'œil, où on doit l'écouter. Celan plonge le langage dans la nuit (image de l'écluse

10. Voir infra et n. 27. 
dans la Rose de personne) pour le faire réentendre, pour en redonner la mémoire quand partout s'étend l'oubli. Et le travail de Celan traducteur est ce qui nous permet de percevoir ce qui dans le travail de l'écriture poétique est déjà traduit et donc, d'une certaine manière, dissimulé.

Ort et Wort, la paronomase souvent signalée indique l'espace traductif, le non-lieu, l'entre-deux de la traduction. Ce que dit un poème de Schneepart (car si la poésie de Celan parle de la/sa poésie, elle parle aussi de la traduction) :

"UNLESBARKEIT dieser /Welt. Alles doppelt./Die starken Uhren/geben der.Spaltstunde recht,/heiser.//Du, in dein Tiefstes geklemmt,/entsteigst dir/für immer. "; "ILLISIBILITÉ de ce/monde. Tout se redouble.//Les solides horloges/justifient l'heure divisée,/d'un son rauque. Toi, coincé au plus profond,/monte hors de toi/pour toujours. " ( $m a t r$.)

Devant l'illisibilité du monde, le redoublement (traductif) est nécessaire. $\AA$ ce redoublement, le temps (essentiel chez Celan) participe : césure de l'heure. Le sujet peut alors sortir de lui-même en se dissociant de lui-même (mouvement habituel chez Celan) pour s'élancer vers le futur, l'à-venir qui est la loi de toute traduction, le " toujours " résonnant aussi dans te titre du poème « DU SEI WIE DU, immer " (dans Lichtzwang) se concluant par "kumi/ori " ( (lève-toi, rayonne »), la parole d'Isaie qui ne sera pas traduite, marquant de son étrangeté l'espace traductif qui est l'espace de l'écriture.

Quand Celan affirme en 1961 : " Je ne crois pas au bilinguisme en poésie [...]. La poésie - c'est le langage dans son unicité fatale " et que Felstiner remarque que son écriture ne respectait pas entièrement le propos, on peut avancer que la traduction - qui n'est pas du bilinguisme - est ce qui permet de sauver le langage de la menace fatale. Entre les langues ou dans la langue. 


\section{Paul Celan, traducteur et poète}

... l'interruption de l'adresse, comme l'adresse même.

(J. Derrida, Schibboleth. Pour Paul Celan)

Il est au demeurant étonnant de constater combien la critique celanienne qui s'appuie tant sur la traduisibilité - ou non - de Celan renvoie rarement à son cuvre traductive (deux tomes, pourtant, sur les cinq des Gesammelte Werke de Suhrkamp). Lorsque John Felstiner (1995) parle de " Translation counterpoint " en donnant ce titre à l'un des chapitres de son livre sur Celan, je choisis alors d'entendre le terme au sens musical. D'autant plus en me souvenant des titres (et de la structure) des deux plus célèbres poèmes de Celan, " Todesfugue ", " Fugue de mort ", et " Engfuhrung ", " Strette ».

Observer le travail traductif de Celan, ses choix, ses procédés, nous introđuit au fonctionnement de la poétique celanienne et permet de comprendre le travail sur la langue qu'est le texte celanien. En effet, si toute poétique est une altération de la langue, c'est-à-dire la production d'une altérité dans le matériau langagier, l'étude des traductions faites par un auteur, dans lesquelles le jeu altératif est essentiel et explicite, offre une perspective privilégiée sur son écriture. Et une telle approche s'impose d'autant plus pertinemment dans le cas de Celan que toute sa poésie est animée par " la question de l'Autre », selon l'expression de M. Broda". Or l'altérité n'est pas tant thématisée dans le texte celanien qu'agissante sur la matière textuelle même, sur la langue, à la façon

11. Voir son article sous ce titre dans Poésie et altérité (1990), pp. 53-59. On ne s'étonnera guère de trouver dans le même recueil un article de H. Meschonnic portant sur « Transformation du traduire et altérité ", pp. 105-115. M. Broda prolonge son analyse autour des themes de l'interlocuteur et du destinataire dans Dans la main de personne. Essai sur Paul Celan (1986). 
d'une inflexion, d'un marquage ${ }^{12}$ : une pulsion la parcourt qui, dans son énonciation, la destine à l'autre.

Cette tension ou torsion de la langue permet d'accueillir l'altérité sans la dissoudre dans un savoir ou une expérience mais en lui laissant l'espace ouvert d'un devenir non médiatisé. Dès lors le processus langagier le plus apte à figurer cette fonction d'altération permanente est celui qui prend place dans un non-lieu, une utopie ${ }^{13}$, l'entre-langues de la traduction. De même que la poésie de Celan est cernée par le silence (le non-lieu de l'innocence textuelle), le silence du début de l'Entretien dans la montagne, de même le risque du silence, selon Benjamin (1971, p. 275), menace et garantit les grandes traductions dont le modèle idéal se situerait dans la spatialité des versions interlinéaires des Écritures.

Ce n'est donc ni un détour ni une digression que de porter un regard sur les traductions de Celan mais un jalon essentiel d'une analyse de sa poétique. La langue de Celan, la langue dont nous parle Celan, par sa position dans l'histoire, est une langue de traduction, fonctionnant comme une traduction. Cette langue sera aussi celle de ses traductions. Une analyse générale de tous les textes traduits, à partir des diverses langues mentionnées, devrait être entreprise pour dégager la poétique de la traduction de Celan et montrer qu'elle ne fait qu'une avec la poétique à l'œuvre dans les poèmes $^{14}$. La multiplicité linguistique des sources, la diversité des

12. Voir J. Derrida (1986) et K. Ziarek (1994).

13. " [...] dans la proximité d'un champ ouvert et libre. Et en dernier lieu dans la proximité de l'utopie. " (1979, p. 80) Territoire de la quête du poème, de l'autre et du sujet, tel que le dessine « Le Méridien ».

14. Il serait nécessaire de procéder à la comparaison de ces deux poétiques avec une troisième, celle du " traduire Celan " pour laquelle le matériau (observations sur la pratique ou analyses théoriques) est abondant. 
auteurs et des époques, loin de poser difficulté, concourent à dégager ce qui fait l'unité du geste traductif, unité certes pas méthodologique puisque les procédés varient, entre les pôles de la littéralité et de l'adaptation, mais davantage principielle : Celan répond à une visée traductive qui l'oblige à varier son mode d'écriture - variation constituant la permanence de son principe traductif ${ }^{15}$, à choisir une visée langagière (l'Intention auf die Sprache de Benjamin) en fonction de la visée de l'original :

Ainsi l'indice historique d'une traduction ne se limite pas à renseigner sur l'état historique de la langue - mais sur l'usage que l'on en fait à un moment donné [...]. Dans la différence de la visée sur la langue, du mode du vouloir dire, qui sépare l'original et la traduction, Benjamin a découvert la légitimité, voire la nécessité de l'acte de traduire, différence grâce à laquelle le problème de la fidélité ou de l'infidélité des traductions est dépourvue de sens. (Szondi, 1982, pp. 147-148)

L'herméneutique traductive répond à la même règle d'historicité que l'herméneutique interprétative et $\mathrm{y}$ fonde la diversité de ses modalités expressives ${ }^{16}$.

Quelques pistes ont été tracées dans l'étude des traductions celaniennes, dont les suivantes. Peter Szondi, dans ses CelanStudien, a consacré l'essai précité à l'analyse de la traduction du sonnet 105 de Shakespeare qu'il envisage dans les termes thériques de Benjamin et à partir de laquelle il dégage une poétique de la constance à l'cuvre dans le texte traduit, et non plus seulement de manière thématique comme dans l'original. Énoncé applicable en fait à toute l'écriture celanienne : « [...] la langue de Celan ne parle

15. Raison pour laquelle Szondi dégage une poétique traductive de Celan à partir du sonnet de Shakespeare portant sur la constance.

16. Une des significations du poème " Mit wechselndem Schlüssel " (" Avec une clé changeante ") du recueil De seuil en seuil. 
pas de quelque chose, mais par elle-même. Elle parle des choses et de la langue par la parole même, par le mode sur lequel elle parle. [...] [U]n poème qui ne traite plus de luj-même, mais qui est ce dont il parle » (Szondi, 1982, pp. 152 et 161). Thomas Pepper revient sur cette traduction et ce que révèle sur lui-même le commentaire de Szondi dans le volume Word Traces (Fioretos, dir., 1993, p. 353) qui contient également un' article de Hans-Jost Frey théorisant la traduction comme métaphore d'elle-même à partir de la version de Celan du sonnet 137 (ibid., p. 345). George Steiner (1978) consacre trois pages (pp. 359-361) de son Après Babel à l'analyse de la traduction du sonnet 79 de Shakespeare pour conclure à une " réciprocité dans la proximité " qui illustre "l'herméneutique de la compensation " au cour de sa théorie de la traduction. C'est également aux traductions de poésie anglaise (Dickinson, Shakespeare, Frost) que John Felstiner (1995, p. 200 sq.) consacre un chapitre de son livre - livrant sa définition : « repetition with a difference - the genetic code of translation » (p. 204) -, à côté de nombreuses autres pages où il traite des traductions du français et $d u$ russe. Martin Bidney (1991, p. 44), enfin, a étudié les « stratégies 》 de traduction de Celan à l'endroit d'Evtouchenko et d'autres poètes russes ${ }^{17}$. Tout en posant qu'il n'y a pas de principes uniques de traduction chez Celan, il repère cependant trois approches récurrentes : variations formelles, amplification ou commentaire, critique de la sensibilité du poète traduit. Il s'agit, dit-il, d'une " compétition de virtuosité " (virtuoso competition) qui rend les traductions de Celan immanquablement reconnaissables comme des textes $^{18}$ de Celan. Adoptant la pertinence de l'image et la

17. Sur la traduction de Mandelstam, voir aussi l'article de L. Olschner dans Word Traces.

18. Texte sera ici à prendre non pas dans une acception neutre et descriptive mais dans celui qu'invoque Meschonnic lorsqu'il parle de " traduction-texte " (voir " Propositions pour une poétique de la traduction ", Pour la poétique $I$, pp. 305-323). Toute traduction doit viser cette authenticité et cette légitimité, car il n'est d'autre fidélité. 
prolongeant, j'ajouterai que dans ses traductions, Celan agit non pas comme un traître (la vieille malédiction attachée au traducteur) mais comme un rival, pour reprendre deux types de duplicité identitaire développés à l'envi au XVII' siècle, les opéras de Mozart par exemple.

Je proposerai à mon tour une analyse partielle sur quatre exemples pour tenter de cerner la poétique traductionnelle de Celan, non en termes de système - rien ne saurait être plus étranger à la pensée de Celan - mais pour en dégager, inspiré par Szondi, ce que Benjamin énonçait comme Art des Meinen. Certes, Benjamin traitait du mode de signifier d'une langue mais il n'est pas abusif, on l'a compris, de considérer une langue-Celan. Non un style ou un idiolecte, mais, propre à tout créateur sujet de et dans son historicité, une visée du monde qui à la fois soutient sa langue et est produite par elle. Barthes la décrivait comme une " écriture », d'autres (dont Meschonnic) la comprennent comme poétique et utilisant ce dernier terme, $j$ 'entends indiquer et expliquer que nulle différence ne distingue à ce niveau, chez Celan comme ailleurs, la poésie de la traduction $^{19}$.

\section{A. Desnos}

\section{LE DERNIER POĖME (Robert Desnos)}

J'ai rêvé tellement fort de toi,

J'ai tellement marché, tellement parlé,

Tellement aimé ton ombre,

Qu'il ne me reste plus rien de toi.

Il me reste d'être l'ombre parmi les ombres

D'être cent fois plus ombre que l'ombre

D'être l'ombre qui viendra et reviendra dans ta vie ensoleillée.

19. "Language is set in motion in many ways, but no relations to language do so more than those named translation and poetry. " D. J. Schmidt (1990), p. 4. 
Vor lauter Von-dir-Trallmen, lauter Gehn, lauter Sprechen mit deinem Schatten, lauter Ihn-Lieben, bleibt mir nun nichts mehr von dir, bleibt mir nur dies : der Schatten Schatten zu sein, der Schatten-Schemen, der ein und aus geht bei deinem sonnigen Leben. (Celan, Gesammelte Werke, t. IV, 1983, pp. 802-803)

LE DERNIER POĖME (Traduction littérale de la traduction de Celan)

De seulement rêver-à-toi, seulement aller, seulement parler avec ton ombre, seulement aimer-elle, ne me reste plus rien de toi, ne me reste que ceci : être l'ombre des ombres, l'ombre-illusion, qui va et vient dans ta vie ensoleillée.

Quelques traits immédiatement repérables ${ }^{20}$ : traitement de la masse et de l'extension typographiques (10 vers au lieu de 7); modification de l'ordre logique des syntagmes signifiants marquée, par exemple, par l'effacement du " je " qui n'apparaît, sous une forme restreinte, qu'au vers 5 ; innovations dans la construction syntaxique et morphologique ("Von-dir-Traümen ", "IhnLieben ") ; enrichissement sonore et sémantique ("Schatten-

20. Le manque de place n'en est pas le seul motif. Voir infra mes considérations sur l'herméneutique du sourcier. 
Schemen 》) ; glissement sémantique (" lauter » qui fait passer le " tellement " vers un " uniquement », de l'intensité à l'unicité) ; changement du temps verbal : le futur du dernier vers qui passe au présent. Sans approfondir notre interprétation, disons succinctement que le poème quitte ainsi le lyrisme romantique de Desnos pour devenir un témoignage exprimé dans l'immanence de l'expérience : l'idéalisme de la passion cède la place au réel d'une souffrance amoureuse ${ }^{21}$.

\section{B. Pessoa (et Nerval)}

Celan traduisit Fernando Pessoa sans attendre les diktats de la mode culturelle et éditoriale. Comment pouvait-il ne pas le faire quand l'un des poèmes traduits " Tabacaria ", "Bureau de tabac ", commence ainsi : « Não sou nada ", « Je ne suis rien ", et que toute la pósie de Celan privilégie les figures du rien et de personne? Extrait de la traduction de ce poème :

[...]

Ou princesa de trovadores, gentilissima e colorida, Ou marquesa do século dezoito, decotada e longínqua, Ou cocote célebre do tempo dos nossos pais, [...]. (Pessoa)

o Furstin der Troubadours, huldvoll und farbengeschmlickt!

o Marquise des Dix-huitième, so fern und so dekolliert!

$O$ vielberuhmte Kokotte aus der Zeit unsrer Văter [...]. (Celan, Gesammelte Werke, t. V, 1983, pp. 586-587)

Les marques du passage traductif sont évidentes : ajout des points d'exclamation ęt des so au deuxième vers; glissement du $O u$ qui aurait suggéré un $O d e r$ au $O$ d'apostrophe, faisant des figures féminines multiples chez Pessoa une figure unique aux diverses incarnations ; surgissement de la troisième langue : «troubadour ",

21. Les variations sur les catégories plus que grammaticales du " je » et du " tu ", notamment, sont essentielles dans l'æruvre de Celan et ont été largement commentées. 
" marquise 》 et surtout « Dix-huitième » alors que cocote et célebre du texte source, mots portugais, appelleraient le français mais apparaissent dans leur version allemande. $\dot{A}$ l'image de la consolatrice venue de " l'Impossible " - "Toi qui consoles, qui n'existes pas et par là même consoles » (Pessoa, 1991, p. 207) - se substitue celle d'une féminité avec laquelle le traducteur/poète entretient un rapport nourri de son propre vécu. Fusion de l'idéal et du réel, ou plutôt déplacements de l'un vers l'autre, que l'on retrouve par le même traitement rhétorique, l'usage de l'apostrophe, dans la traduction des "Cydalises » de Nerval :

O pâle fiancée $1 / O$ jeune vierge en fleur $1 /$ Amante

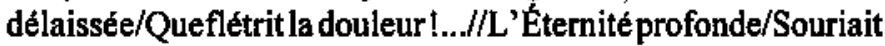
dans vos yeux [...].

O Bleiche, mir verlobte !/O Jungfrau kaum erbluht !/Ich liess dich deiner Liebe,/am Schmerz bist du verglüht !//O Ewigkeit, o tiefe,/die aus dem Aug dir sprach. [...] (Gesammelte Werke, t. IV, pp. 806-807)

En même temps que le rapport amoureux se personnalise par l'usage de la première personne du singulier, la fiancée et l'éternité sont regroupées dans la même adresse.

\section{E. Dickinson}

Les deux premiers quatrains d'un célèbre poème :

Because I could not stop for Death He kindly stopped for me The Carriage held but just Ourselves And immortality.

We slowly drove - He knew no haste And I had put away My labor and my leisure too, For his Civility [...] 
DER TOD, da ich nicht halten konnt, hielt an, war gern bereit.

Im Fuhrwerk sass nun er und ich und die Unsterblichkeit.

Ihm gings auch langsam schell genug, und ich hatt fortgetan das Fronen und das Müssiggehn, so freundlich war der Mann.

[...] (Celan, Gesammelte Werke, t. V, pp. 382-383)

La mort, que je ne pouvais arrêter, s'est arrêtée, elle y était tout à fait disposée.

Dans la charrette se sont assis rien qu'elle [lui en allemand] et moi et l'immortalité.

Il lui convenait aussi d'aller lentement suffisamment vite et je m'étais débarrassé

de la corvée et de l'oisiveté tant aimable était l'homme.

[...] (Traduction littérale de la traduction de Celan)

Sans nous arrêter aux détails, le sens des modifications (syntaxiques, lexicales, génériques, typographiques) est explicite et elles convergent à donner à la figure de la mort un effet dramatiquement (au sens théâtral) plus concret et présent. Ce que dit Feistiner dans son commentaire de la traduction d'un autre poème de Dickinson s'applique parfaitement à celui-ci : " It takes translation, Celan's voice superimposed upon the earlier poet's, to get a disillusioned sense of twentieth-century death. 》 (1995, p. 205) Si Celan partage avec l'auteur anglais du XIX siècle une détresse hantée par la mort et la solitude, il en transmet la teneur au lecteur par une personnification et une mise en scène qui dépouillent la poésie de Dickinson de ses éléments diaphanes et abstraits pour en extraire la charge affective et existentielle.

S'il n'était hasardeux de tirer une conclusion à partir d'un corpus aussi mince, je dirais que ces trois exemples participent d'un même travail de transformation sur l'original : le tirer vers un 
surplus de réalité ${ }^{22}$, le lester d'une concrétude qui correspond chez Celan au refus d'admettre d'autres plans d'existence et d'expérience que la matérialité et l'histoire et à l'impossibilité de penser ce vécu dénudé ailleurs que dans le langage. Celan écrit dans son aujourd'hui - qui n'est que d'être " après Auschwitz " - et en affronte les tourments et les doutes dans une lucidité haletante. Ne reste à l'homme que les mots pour dire les choses et si celles-ci menacent de leur poids et de leur tranchant acéré, les mots en traduiront la violence et l'écartèlement, en épouseront la ténèbre et le dénuement. On est loin du territoire abstrait ou irréel que certains critiques ont cru reconnaître dans la poésie celanienne. La nuit de Celan n'est pas une obscurité métaphysique, le vide qui guette et cerne sa parole n'est pas un silence ontologique, son destin n'est pas celui de Holderlin. Écrire dans l'aujourd'hui parce qu'Auschwitz a ruiné toute possibilité d'écrire dans une continuité en fracassant l'ontologie d'une temporalité linéaire où les événements se succèdent selon une logique dont la métaphysique de la tradition occidentale assurerait le fondement. Tout un réseau métaphorique évoqué supra exprime chez Celan la scission ou césure chronologique : Schrunde der Zeit, crevasse du temps, et inmitten spliternde Stunde, parmi les heures fendillées, ( Vor einer Kerze », Von Schwelle zu Schwelle), Zeitenschrunde, crevasse du temps ("Weggebeitz ", Atemwende), Stundenzäsur, césure des heures ("Und mir dem Buch aus Tarussa », Die Niemandsrose),

22. "Les poèmes [...] font route vers quelque chose./Vers quoi? Vers quelque lieu ouvert, à investir, vers un toi invocable, vers une réalité à invoquer. " (" Discours de Brême ", 1987, p. 17) Un principe de réalité, instance de référence ou visée de quête, anime constamment la pensée de Celan et le thème apparaît récuremment dans ses textes théoriques (la réponse au questionnaire de la librairie Flicker de 1958, par exemple) ou sa correspondance. Ce souci est lié à son insistance, exprimée à plusieurs reprises, sur la nature non hermétique de sa poésie. Felstiner l'explique avec justesse par la déresponsabilisation du lecteur qu'un tel hermétisme impliquerait et permettrait (op. cit., p. 253). 
Spaltstunde, heure scindée ( Unlessbarkeit 》, Schneepart), Narbe der Zeit, cicatrice du temps ("Abend der Worte », Von Schwelle zu Schwelle) ou encore le titre du recueil Zeitgehöft, Enclos du temps. Ainsi peut-on comprendre - et accepter - l'affirmation d'Adorno (1986), modulée par la suite mais tant débattue, selon laquelle " écrire un poème après Auschwitz est barbare. " Écrire dans une logique temporelle, une chrono-logie, qui supposerait la possibilité d'un après, d'une postériorité (comprise aussi comme postérité) à Auschwitz, d'une histoire qui aurait continué sans en être transformée, telle serait l'attitude que toute décente éthique ne peut que violemment refuser. Le pessimisme méthodologique d'Adorno dont la radicalité est indispensable à repenser l'ontologique épouse l'historicité de son exercice et la conscience de sa situation :

Karl Krauss a bien fait d'intituler sa tragédie les Derniers jours de l'Humanité - ce qui se passe maintenant mériterait de s'appeler Après la fin du monde. [...] L'idée qu'après cette guerre la vie pourrait continuer 'normalement' ou même qu'il pourrait y avoir une 'reconstruction' de la civilisation (Kultur) - comme si la reconstruction de la civilisation n'en était pas déjà en elle-même la négation - est une idée stupide. (1983, pp. 52-53)

C'est dans cette perspective que la traduction comme transformation $^{23}$ prend tout son sens. Dynamique qui éclaire de même le lien établissant une poétique commune à l'écriture et à la traduction. Il nous suffira ici pour le poser de rapprocher la définition de Meschonnic de la traduction comme « rapport demeuré rapport » de la suivante de Char : « Le poème est l'amour réalisé du désir demeuré désir » (" Partage formel » dans Seuls demeurent). Poésie et traduction transforment le langage comme ils transforment le sujet, auteur ou lecteur, saisi dans cette opération. Pour l'une et

23. On trouve telle théorisation dans les positions du collectif Change (1973), les analyses de Jacques Derrida (voir notamment Positions, 1972, p. 31) ainsi que chez Michel Serres (1974). 
l'autre, le sens de l'énoncé tient dans l'énonciation et non dans une signification autonome et extérieure, il est à trouver dans le mouvement même du langage ${ }^{24}$, dans le devenir qu'il implique et qu'il manifeste. La phénoménologie de la traduction s'expérimente comme transformation et cette vision nous invite à abandonner les idées de parcours et même de passage, habituellement utilisées (ce dont témoignent les notions de départ et d'arrivée pour désigner les langues ou les textes en jeu), ainsi qu'à réviser les raisonnements fondés sur des postulats d'équivalence, de compensation ou de fidélité. Walter Benjamin écrivait précisément : « La traduction est le transfert d'une langue dans une autre à travers un continuum de métamorphoses. La traduction traverse des continuums de métamorphose, non pas des zones abstraites d'équivalence et de ressemblance. " (Benjamin, 1966, p. $20 ;$ ma trad.) ${ }^{25}$ Et dans son autre texte majeur sur langage et traduction, " la Tâche du traducteur », Benjamin avance significativement son postulat : « La traduction est une forme ", à la suite du développement sur la fonction non-communicatrice de la la traduction ${ }^{26}$.

24. Pour Celan le poème est " événement, mouvement, marche, [...] tentative de trouver une direction " (" Discours de Brême ", Poèmes (1987a), p. 16.

25. « Die Obersetzung ist die Oberführung der einen Sprache in die andere durch ein Kontinuum vonVerwandlungen. Kontinua der Verwandlung, nicht abstrakte Gleichheits- und Ähnlichkeitsbezirke durchmi t die Übersetzung. " (1966), p. 20. C'est parce que la traduction est transformation, champ de résonnance que traversent toutes sortes de textualités - ce que dit précisément Benjamin - que j'ai employé les termes de " transfert " et de " métamorphose " en écho à leur emploi chez Freud et Kafka.

26. "Mais ce que contient un poème hors de la communicationet même le mauvais traducteur conviendra que c'est l'essentiel - n'est-il pas universellement tenu pour l'insaisissable, le mystérieux, le 'poétique'?" (1971, pp. 261-262) 
Celan traducteur ne répond pas à une fonction communicatrice puisqu'il prend dans le poème ce qui en fait lui permet d'en écrire un autre. Il ne cherche pas à reproduire l'intégrité du poème original mais à (re)constituer une cohérence à partir d'éléments transformables. Appropriation certes, dans le sens de donner à l'original une propriété, une identité autre qu'il contenait mais que le poète avait choisi de taire au profit d'une " formesens » (Meschonnic) différente. Là se tient la richesse d'une littéralité - non d'un littéralisme, qui est une notion idéologique comme visée productrice : la forme autre était en puissance et en attente $^{27}$ et se réalise dans un autre aujourd'hui. Traduction fidèle parce qu'infidèle : l'historicité et non l'historicisme, c'est-à-dire le dialogue entre présents sucessifs, moments pareillement chargés ou investis de " Jetztzeit », pour reprendre le lexique de la philosophie de l'histoire benjaminienne.

" Abend der Worte - Rutengänger im Stillen! » ; "Soir des mots - sourcier dans le silence ! " (1991, p. 75) Le vers de Celan me permet de théoriser cette poétique traductive en renouvelant une figure symbolique quelque peu surexploitée en traductologie. Dans le processus traductif, l'acte premier de lecture et de réception s'apparente non à un éloignement du texte de départ (ce que suggérerait justement ce terme idéologiquement chargé) mais à un obscurcissement ${ }^{28}$ et, d'une certaine manière, à une réduction au silence des mots de l'original afin de dégager leur signifiance de leur signification, retrouver dans leur dit la force et la fertilité de

27. Ce qu'exprime le thème messianique dans la pensée du langage et de la traduction chez Benjamin.

28. Voir mon article « L'Obscurcissement. Lecture d'un motif dans l'œuvre de Paul Celan " à paraître dans Frontières (Montréal), "Littérature et mort ". 
leur dire ${ }^{29}$. Non pas remonter ou revenir au mythique niveau d'un logos primordial, ce serait faire l'économie d'une indispensable historicité de l'énonciation, mais se mettre à l'écoute de tous les échos possibles de l'énoncé, une écoute qui tiendrait à la fois de la réduction phénoménologique et de l'attention herméneutique. Là où la rationalité classique comprend l'intelligibilité en termes de clarté, une raison traductive inspirée du texte celanien suggérerait une vérité intégrant le sombre et l'inaudible, acquiesçant à l'ombre et au murmure. Là où le puisatier creuse afin de faire jaillir un flot attendu, le sourcier ${ }^{30}$ se contente d'indiquer le lieu d'un possible jaillissement. Le traducteur n'a ni la fonction ni la responsabilité d'extraire de la gangue textuelle un joyau sémantique brillant d'un éclat définitif. Son geste est davantage proche de celui du peintre impressionniste cherchant à rendre le mystère de la lumière sans le percer, sans le trahir. Trahison s'en suivra cependant, puisque passé ce stade de compréhension, le traducteur doit choisir un énoncé en accord avec son historicité singulière, mais trahison contingente, nécessaire, assumée, responsable, exercice d'une liberté non dominatrice majs garantie par l'écoute première de l'autre".

Celan s'approprie le texte de l'autre comme il a dâ s'approprier une langue devenue celle de l'autre. Le second transfert, suscité par l'histoire, autorise et éclaire le premier. Au

29. L'emploi de ces deux termes se veut un renvoi à la philosophie lévinasienne mais dans une stratégie rhétorique d'un effet et d'une ambition bien moindre que leur déploiement chez l'auteur d'Autrement qu'être, ou au-delà de l'essence.

30. Tel est le trope redessiné - et revalorisé positivement, le sourcier du couple sourcier/cibliste thématisé par Jean-René Ladmiral et ayant connu le succès que l'on sait. Voir mon article (1995).

31. On reconnaîtra aisément ici les marques de la pensée de Lévinas sur la subjectivité vue comme sub-jection à autrui. 
lecteur (lecteur, critique ou traducteur) de faire de même $\mathrm{e}^{32}$. Le processus d'enrichissement, " compensation " ou " restitution " selon les termes de Steiner, opère pleinement ici et démontre combien et comment la lecture ou la traduction d'un poème ne peuvent être qu'un autre poème, loi de l'authentique rencontre, que seul le sujet dans son histoire saura respecter puisqu'il est garant de sa légitimité, gardien de la loi qui le garde. Ars translatandi ou ars interpretandi qu'énonce pleinement exprimé le poème « Mit wechselndem Schlüssel ", " D'une clé qui change » :

D'une clé qui change,/tu ouvres la maison oùttournoie la neige des choses tues./Au gré du sang qui sourd/de ton oreille ou ton œil ou ta bouche,/ta clé change./Ta clé change, le mot change,/qui peut partager la course des flocons./Au gré du vent qui te repousse/la neige se roule autour du mot. (1991, p. 65)

\section{Conclusion : La fosse de Babel}

"Und es steigt eine Erde herauf, die unsre,/diese./Und wie schicken/keinen der Unsern hinunter/zu dir,/Babel. "; " Et monte une terre, la nôtre,/celle-ci./Et nous n'envoyons/aucun des nôtres en bas,/vers toi,/Babel. » (1979, p. 117)

Étrange spatialité. Inversion de la verticalité. La terre s'élève et Babel, métonymiquement pris pour la Tour de Babel, se situe en bas. Mais un renvoi intertextuel nous rappelle un aphorisme

32. A noter un phénomène éditorial qui n'apparaît pas mineur et qui pourrait être considéré en corrélation avec la poétique celanienne: les deux tomes de ses traductions dans les Fuvres complètes chez Suhrkamp sont bilingues, de même que la majorité des traductions de ses poèmes en français ou en anglais. Le texte de départ systématiquementen regard du texte d'arrivée: pour reprendre un jeu de mots cher à Lévinas, l'un regarde l'autre, au sens de l'un concerne l'autre (" ça le regarde $»)$. 
de Kafka : "Nous creusons la fosse de Babel ${ }^{33}$." (1980, p. 334) Énoncé qui nous renvoie à son tour à Celan, au tout premier poème du recueil, circularité herméneutique entre Celan et Kafka, et entre les poèmes à l'intérieur même de la Rose de personne $e^{34}$, le précité figurant dans la quatrième et dernière partie du recueil. Le premier poème donc qui dit :

" ES WAR ERDE IN IHNEN, und/sie gruben./Sie gruben und gruben, so ging/ihr Tag dahin, ihre Nacht. [...] Sie gruben und hörten nichts mehr;/sie wurden nicht weise, erfanden kein Lied,/erdachten sich keinerlei Sprache./Sie gruben. " ; "IL Y AVAIT DE LA TERRE EN EUX, et/ils creusaient.//ls creusaient, creusaient, ainsi/passa leur jour, leur nuit. [...] lls creusaient et n'entendaient plus rien ;/ ils ne devinrent pas sages, $n$ 'inventèrent pas de chanson, $/ n$ 'imaginèrentaucunesorte de langue./Ils creusaient. " (lbid., p. 13)

Spatialité inversée du haut et du bas, du ciel et de la terre ? La premier poème parle de la terre, le dernier du recueil s'intitule "In der Luft », " En l'air ». Parcours salvateur de la terre au ciel qui serait le cheminement du recueil et l'expérience de sa poétique, pour le poète comme pour le lecteur ? Outre que l'univers celanien ne suppose aucune rédemption puisque façonné de cendres et de silence, avec le travail sur la langue comme seule voie d'accès au réel, le dernier poème indique précisément : " IN DER $L U F T$, da bleibt deine Wurzel, da,/in der Luft.Wo sich das Irdische ballt, erdig,/Atem-und-Lehm. " ; " EN L'AIR, là reste ta racine, là,/en l'air./Où le terrestre se ramasse, terreux,/Souffle-et-Limon. " (Ibid., p. 153) Et la " Fugue de mort »: " wir schaufeln ein Grab in den

33. Le sens de l'aphorisme se confirme d'un second: " S'il avait été possible de bâtir la tour de Babel sans l'escalader, cela aurait été permis » (ibid.), p. 76.

34. Procédé habituel chez Celan dont la construction des recueils est en soi un effet d'écriture et de sens. 
Lüften da liegt man nicht eng "; " nous creusons une tombe dans les airs on n'y est pas couché à l'étroit " $(1987$, p. 85)

Non tant, donc, un renversement des catégories qui respecterait encore l'intelligibilité de leur organisation en un système mais leur brouillage, mesure du réel dont témoigne Celan en notre histoire, qui, par constraste, fait ressortir l'importance du mouvement, du geste, ici le creusement ${ }^{35}$. La Tour de Babel située vers le bas (Celan), creusée (Kafka) : que faut-il ici entendre ${ }^{36}$ ? Il y a mouvement ${ }^{37}$, celui de la Tour, celui que donnaient les bâtisseurs de la Tour, mais mouvement purifié, réduit à son intention: montée vers le bas, creusement dans l'aérien. Les bâtisseurs de Babel voulaient " se faire un nom " par désir d'unité et le résultat fut la dispersion. Comment préserver le(s) nom(s) ${ }^{38}$ ? Comment conserver le langage en sa faculté d'assurer l'humanité de l'humain en une histoire qui a accueilli sa radicale négation, son effacement ? Chez Kafka, les noms ne s'élèvent plus, ne se déclinent plus dans la manifestation, l'explicitation, mais se retrouvent enfouis, enterrés, secrets et ainsi sauvés. Et de Celan Lévinas (1987) dit, dans un texte du recueil Noms propres, que sa poésie s'écrit

35. À noter le thème essentiel, principiel, chez Celan de la profondeur, encore plus accentué de ce doute posé sur l'essence de l'espace, dont l'ordonnance est soupçonnée au même titre que le temps chronologique et linéaire. Voir le poème « Das Wort vom Zur-Tiefe-gehen ", « Le mot d'aller-àla-profondeur " $(1979$, p. 15).

36. Entendre et non comprendre: comme chez Benjamin, l'historicité nous impose une herméneutique de l'« illumination " plus que de l'intellection.

37. Notion essentielle, on le sait, chez Celan qui lui sert notamment à définir le poème.

38. Voir sur cette question notre article, " Noms secrets et noms sacrés » dans le Texte et le nom, à paraître aux Éditions XYZ (Montréal). 
dans une " langue du neutre " qu'il définit ainsi : « Langage de la proximité pour la proximité, plus ancien que la vérité de l'être - que probablement il porte et suppporte - le premier des langages, réponse précédant la question, responsabilité pour le prochain, rendant possible, par son pour l'autre toute la merveille du donner. " (p. 50) On donne un nom, on donne la parole, comme on donne la vie, et de même que la vie serait ce don même, la vérité du langage tiendrait dans sa nature de langage. Un langage qui ne se présente pas dans la communication, l'économie de l'échange et de la relation mais dans une pureté qui le précède, au plus près de la reine Sprache de Benjamin ou de sa « langue des noms " à l'origine de l'origine, au plus près de la traduction qui pour Benjamin n'a fonction que de révéler la nature de langage des langues, au plus près encore du " Dire " lévinassien dont le signe ne signifie que la signification, que la donation du signe. Similaire au mouvement qui, sans direction, ne tire son sens que de lui-même, le langage ne transmet que le sens de son exercice. Parler pour parler, " parler pour ne rien dire ${ }^{39}$ " (Lévinas, 1990, p 225), parler sans dire mot : dignité d'une humanité qui ainsi ne serait pas complice du monde de réification et d'aliénation qui désormais l'abrite. Le langage refuse l'élévation vers la clarté diurne et aveuglante de la signification mensongère. "HURIGES SONST. Und die Ewigkeit/blutschwarz umbabelt. "; "PROSTITUTION DE L'ALTERNATIVE. Et l'éternité/embabelée de noir-sang. " (Schneepart; ma trad. ${ }^{49}$ Mensonge de tout autre énoncé : vérité de la vie (l'éternité) si on lui accorde sa charge de ténèbre (noir-

39. Lorsque Lévinas présente le dire comme signifiant « 'me voici' [...] sans avoir rien à quoi m'identifier, sinon au son de ma voix ou à la figure de mon geste " (ibid., p. 233), on ne peut que songer au poème comme poignée de mains de Celan (cf. aussi Benjamin, 1971, p. 261, et notre commentaire de la page 23).

40. "Embabelée »: je m'autorise ici de la traduction de $\mathrm{H}$. Meschonnic du passage biblique sur la Tour de Babel dans les Tours de Babel (1985), p. 13. 
sang), si on lui reconnaît l'horreur dont elle est désormais et à jamais affectée, vérité alors d'un tel dire dont Babel - langue de l'histoire et histoire de la langue - témoigne en abandonnant la majuscule du mythos pour se fondre dans le cours d'un logos simplement humain, dans le réel adjectival d'une parole commune.

L'écriture comme survie. Benjamin n'appelait pas différemment la traduction. Nous sommes les survivants de Babel : ceux qui survivent à sa ruine, ceux qui en perpétuent l'héritage.

\section{Références primaires : Paul Celan}

La Rose de personne (1979a). Paris, Le Nouveau Commerce, tr. M. Broda.

" Le Méridien " (1979b). Po\&sie, n 9, tr. J. Launay.

Enclos du temps (1985). Paris, Clivages, tr. M. Broda.

Poèmes (1987a). Le Muy, Éditions Unes, tr. J. E. Jackson.

Pavot et mémoire (1987b). Paris, Christian Bourgois, tr. V. Briet.

Contrainte de lumière (1989). Paris, Belin, tr. B. Badiou et J.-C. Rambach.

Entretien dans la montagne (1990a). Paris, Éd. Michel Chandeigne, tr. S. Mosès.

Strette et Autres Poèmes (1990b). Paris, Mercure de France, tr. J. Daive.

De seuil en seuil (1991). Paris, Christian Bourgois, tr. V. Briet.

Grille de parole (1993). Paris, Christian Bourgois, tr. M. Broda. 


\section{Autres recueils cités :}

Atemwende, Fadensonnen, Lichtzwang, voir Gesammelte Werke in funf Bänden, Frankfurt am Main, Suhrkamp, 1983.

\section{Références secondaires}

ADORNO, T. W. (1983). Minima Moralia. Réflexions sur la vie mutilée (Paris, Payot, 1983 ; tr. E. Kaufholz et J.-R. Ladmiral)

(1986). Prismes. Critique de la culture et société. Paris, Payot ; tr. G. et R. Rochlitz).

(1989). Théorie esthétique. Paris, Klincksieck ; tr. M.

Jimenez).

BENJAMIN, Walther (1966). " Uber die Sprache Uberhaupt und uber die Sprache des Menschen " [Sur le langage en général et sur le langage humain], Angelus Novus, Ausgewdihle Schriften 2, Frankfurt am Main, Suhrkamp Taschenbuch.

(1971). " La Tâche du traducteur », CEuvres I, Mythe et violence. Paris, Denoêl ; tr. M. de Gandillac).

BIDNEY, Martin (1991). « Paradoxical Homage: Celan's strategies for translating Evtushenko and other Russian poets ", in BLOCK, H. M., dir. (1991).

BLANCHOT, Maurice (1988). L'Espace littéraire. Paris, Gallimard, Folio/Essais.

BLOCK, H. M., dir. (1991). The Poetry of Paul Celan. New York, Peter Lang.

BRODA, M. (1986). Dans la main de personne. Essai sur Paul Celan. Paris, Cerf.

Collectif Change (1973), 14, février. Paris, Seghers/Laffont. 
DELEUZE, Gilles (1993). Critique et clinique. Paris, Minuit.

DERRIDA, Jacques (1972). Positions. Paris, Minuit. (1986). Schibboleth. Paris, Galilée.

FELSTINER, J. (1995). Paul Celan: Poet, Survivor, Jew. New Haven et Londres, Yale University Press.

FIORETOS, A. dir. (1993). Word Traces. Readings of Paul Celan. Baltimore et Londres, The John Hopkins University Press.

JABĖS, Edmond (1990). La Mémoire des mots. Comment je lis Paul Celan. Éd. Fourbis.

KAFKA, Franz (1980). "Cahiers divers et feuilles volantes ", Préparatifs de noce à la campagne. Paris, Gallimard ; tr. M. Robert.

LÉVINAS, Emmanuel (1987). Noms propres. Paris, Le Livre de poche, Biblio/Essais.

(1990). Autrement qu'être, ou au-delà del'essence.Paris, Le Livre de poche, Biblio/Essais.

MESCHONNIC, Henri (1985). Les Tours de Babel. Mauzevin, TransEurop-Repress.

(1986). Pour la poétique II. Paris, Gallimard,

(1990). La Rime et la vie. Lagrasse, Verdier.

NOUSS, Alexis (1995a). " La Traduction comme OVNI ", Meta, XL-3, septembre.

(1995b). La Modernité. Paris, P.U.F., coll. «Que saisje?».

PESSOA, Fernando (1991). Le Gardeur de troupeaux. Paris, Gallimard, coll. «Poésie» ; tr. Armand Guibert. 
PROUST, Marcel (1971). Contre Sainte-Beuve. Paris, Gallimard, La Pléiade.

ROBIN, Régine (1993). Le Deuil de l'origine. Une langue en trop, la langue en moins. Presses de l'Université de Vincennes.

SARTRE, Jean-Paul (1972). Les Mots. Paris, Gallimard, Folio.

SCHMIDT, D. J. (1990). "Hermeneutics and the Poetic Motion", Hermeneutics and the Poetic Motion. New York, State University of New York at Binghamton.

SERRES, Michel (1974). La Traduction. Paris, Minuit.

STEINER, George (1978). Après Babel. Une poétique du dire et de la traduction. Paris, Albin Michel ; tr. L. Lotringer.

Moscovici), L'Écrit du temps, 14/15.

(1987). " La Longue vie de la métaphore» (tr. M.

SZONDI, Peter (1982). " Poésie et poétique de la constance. Sur la traduction allemande de Paul Celan du sonnet 105 de Shakespeare ", Poésies et poétiques de la modernité. Lille, Presses Universitaires de Lille.

ZIAREK, K. (1994). Inflected Language. Towards a Hermeneutics of Nearness. Albany, State University of New York Press.

RÉSUMÉ : Dans la ruine de Babel : poésie et traduction chez Paul Celan - Paul Celan, l'un des plus grands poètes de langue allemande du $\mathrm{XX}^{e}$ siècle, en est aussi l'un des plus grands traducteurs. Sur les cinq tomes de ses $Q$ Euvres complètes chez Suhrkamp, deux volumes sont onsacrés à ses traductions, en majorité de la poésie, couvrant sept langues et près d'une cinquantaine d'auteurs. Le présent article tente de montrer qu'une même poétique anime les deux gestes d'écriture et de traduction et qu'une telle entreprise doit se comprendre par sa dimension éthique. Il s'agit pour Celan de recréer un langage humain, 
ouvert à l'altérité, répondant à cette sombre historicité qui est de vivre après Auschwitz.

\begin{abstract}
In Babel's ruin : Poetry and Translation in Paul Celan - Paul Celan, one of the greatest German language poets of the $\mathrm{XXth}$ century, is also one of its greatest translators. In the five-volume current edition of his Complete Works (Suhrkamp) two volumes contain his translations, mostly of poetry, from seven languages and of about fifty authors. This article attempts to show that the same poetics underlies both the act of writing and that of translating, and that this double production was Celan's answer to an ethical challenge : to recreate a human language, open to alterity, in the shadow of the specific historicity of living after Auschwitz.
\end{abstract}

Alexis Nouss : Département de linguistique et traduction,
Université de Montréal, C.P. 6128, Succ. Centre-ville, Montréal
(Québec) H3C 3J7 CANADA 